\title{
EVALUACIÓN DE LAS OTOEMISIONES ACÚSTICAS EN RELACIÓN A LOS POTENCIALES EVOCADOS AUDITIVOS DE TRONCO CEREBRAL EN NIÑOS
}

\author{
Julia Rado-Triveño',a, Jaime Alen-Ayca²,b
}

\begin{abstract}
RESUMEN
Con el objetivo de determinar la validez del uso de la otoemisiones acústicas en comparación con los potenciales evocados auditivos de tronco cerebral (PEATC), se realizó un estudio con 96 niños entre cero a cuatro años que acudieron al Instituto Nacional de Rehabilitación en Lima, Perú. Los resultados muestran un punto de corte que corresponde a 1 en ambos oídos, con valores de likelihood ratio (LR) (+): 17,67 en OD y 16,72 en oído izquierdo, y de LR (-): 0,25 en oído derecho y 0,24 en oído izquierdo; se obtuvo Curva ROC con área bajo la curva en oído derecho de $0,830(p<0,001)$ y en oído izquierdo de $0,829(p<0,001)$. Según los resultados de LR (+) la sensibilidad es de $76 \%$ en el oído derecho y de $65 \%$ en el oído izquierdo que coincide con la conformación de la curva ROC. En conclusión, las otoemisiones acústicas no representarían una alternativa lo suficientemente discriminatoria como prueba de tamizaje en esta población.
\end{abstract}

Palabras clave: Pérdida auditiva, Sensibilidad, Especificidad (fuente: DeCS BIREME)

\section{EVALUATION OF OTOACOUSTIC EMISSIONS IN RELATION TO BRAINSTEM EVOKED AUDITORY POTENTIALS IN CHILDREN}

\begin{abstract}
To determine the validity of the use of acoustic otoacoustic emissions in comparison with the evoked potentials Auditory brainstem examination (PEATC), a study was carried out with 96 children between 0 and 4 years of age that went to Instituto Nacional de Rehabilitación in Lima, Peru. The results show a cut-off point corresponding to 1 in (+): 17.67 in right ear and 16.72 in left ear, and LR (-): 0.25 in ear right and 0.24 in left ear; ROC curve with area under the right ear curve of 0.830 $(p<0.001)$ was obtained and in left ear of $0.829(p<0.001)$. According to the results of $L R(+)$ the sensitivity is $76 \%$ in the right ear and $65 \%$ In the left ear that coincides with the conformation of the ROC curve. In conclusion, acoustic emissions would not represent an alternative sufficiently discriminatory alternative as a screening test in this population.
\end{abstract}

Key words: Hearing loss, Sensitivity, Specificity (source: MeSH NLM)

\section{INTRODUCCIÓN}

El tamizaje auditivo neonatal para la detección precoz de hipoacusia, requiere la presencia de la validez concurrente entre la prueba de otoemisiones acústicas y los potenciales evocados auditivos de tronco cerebral (PEATC); estudios previos correlacionaron ambas pruebas encontrando un resultado del $70 \%$ (1). Se obtuvo una correlación totalmente alterada en aquellos que presentaron fallo en las emisiones otoacústicas en ambos oídos, con ausencia de registro de los PEATC. Así mismo, otros estudios no encontraron ningún caso considerado normal en otoemisiones acústicas en niños con sordera profunda o grave con los PEATC (2).
En el estudio realizado por Yousefi et al. (3) se evaluó la sensibilidad y especificidad de las otoemisiones acústicas frente a los PEATC como método de screening auditivo, encontrando que las otoemisiones acústicas presentaban una sensibilidad de $66,7 \%$ y especificidad de $98,8 \%$ para la detección de pérdida auditiva; mientras que Zivié (4), en su estudio, encuentra una sensibilidad de $98 \%$ para las otoemisiones acústicas como método de screening auditivo neonatal.

En objetivo del presente estudio fue determinar la validez del uso de las otoemisiones acústicas frente a los resultados obtenidos en los PEATC; como herramienta de evaluación de la audición en niños, con la finalidad de su ingreso a un programa para diagnóstico definitivo.

\footnotetext{
Instituto Nacional de Rehabilitación “Dra. Adriana Rebaza Flores” Amistad Perú - Japón. Lima, Perú (INR)

Pontificia Universidad Católica del Perú. Lima, Perú.

Magíster en Educación Mención Trastornos de la Comunicación Humana; ${ }^{b}$ médico rehabilitador.

Recibido: 10/12/2015 Aprobado: 07/09/2016
}

Citar como: Rado-Triveño J, Alen-Ayca J. Evaluación de las otoemisiones acústicas en relación a los potenciales evocados auditivos de tronco cerebral en niños. Rev Peru Med Exp Salud Publica. 2016;33(4):706-12. doi: 10.17843/rpmesp.2016.334.2555 


\section{MATERIALES Y MÉTODOS}

\section{DISEÑO Y POBLACIÓN DE ESTUDIO}

Se realizó un estudio observacional, transversal entre los meses de agosto y noviembre de 2012 en el cual se realizaron pruebas de otoemisiones acústicas y PEATC.

La muestra fue no probabilística por conveniencia, que consistió en captar al total de pacientes de cero a cuatro años que asistieron al Instituto Nacional de Rehabilitación (INR) en Lima Perú, derivados para descartar alguna alteración auditiva. Se excluyeron aquellos pacientes con datos incompletos y con antecedente de hiperbilirrubinemia.

\section{INSTRUMENTO}

Para la ejecución de las pruebas auditivas se utilizó el equipo de PEATC ECLIPSE Marca Interacoustics, versión de software $80701264,06 / 11{ }^{(5)}$ y el equipo de emisiones otoacústicas transientes marca OTOREAD, versión de software, año $2011^{(6)}$.

\section{PROCEDIMIENTOS}

El día de la cita el niño debía acudir trasnochado, en ayunas y con los conductos auditivos permeables, según otoscopia previamente realizada. El grado de hipoacusia se determinó tomando en cuenta la mínima intensidad a la que fue identificable la onda $V$ en los registros de PEATC, utilizando la clasificación de hipoacusia avalada por la OMS.

El día de la cita, según el peso del niño, se le administra hidrato de cloral $(50 \mathrm{mg} / \mathrm{kg}$ de peso); cuando el paciente se quedaba dormido, se le recostó en la camilla, se limpió con bencina y alcohol yodado la piel sobre la que se colocaron cuatro electrodos de superficie en el vertex, en el tercio superior de pómulo derecho (1), mastoides ${ }^{(2)} y$, además, se colocan los tips de inserción en cada conducto auditivo externo; se verifica la correcta colocación de los electrodos en el preamplificador y se procede a colocar la intensidad en $\mathrm{dB}$ de los estímulos que fueron de 2000 $\mathrm{Hz}$ tanto a $30 \mathrm{~dB}$ como a $60 \mathrm{~dB}$. Luego del registro gráfico de las curvas, se procedió a ubicar la onda $V$ en todas las intensidades. Se consideró normal el PEATC cuando la onda $V$ estuvo presente a nivel de $30 \mathrm{~dB}$. La duración de este procedimiento es de $30 \mathrm{~min}{ }^{(5)}$.

Las otoemisiones acústicas se realizaron al término de los PEATC, previa preparación del ambiente en condiciones de silencio. Se evaluó cada oído utilizando la sonda del equipo (emisor) marca Otoread, con el tamaño de tip correspondiente según la edad del paciente, que se coloca en el conducto auditivo externo. En base a los parámetros fijados del instrumento, la presencia de una "P" indica que la relación señal-ruido para la prueba, es igual o superior al criterio del instrumento; en el caso de las otoemisiones acústicas, será de $4 \mathrm{~dB}$ y según el número total de "P" obtenidas en la prueba, se considerará: normal (pasa) cuando se obtienen $3 P$ o más, y anormal (a control) en el caso de $1 \mathrm{P} \circ 2 \mathrm{P}{ }^{(6)}$. Según Aguilar ${ }^{(7)}$ las posibilidades de resultado fueron dos: "pasar" o resultado normal (presencia de emisiones otoacústicas en más de dos de las cuatro frecuencias evaluadas) es decir 3P a 4P, y el resultado fue anormal o "a control", en el caso de ausencia de emisiones en dos o más de las cuatro frecuencias evaluadas, es decir, $1 \mathrm{P}$ a $2 \mathrm{P}$.

\section{ANÁLISIS ESTADÍSTICO}

Se describieron la frecuencia, los porcentajes, los promedios, la mediana, y la desviación estándar. Para la obtención de los coeficientes de probabilidad (positivo y negativo), y la curva ROC para determinar el punto de corte en el que se alcanza la sensibilidad y especificidad más alta, tanto para LR (+) y LR (-), cuyos valores mayores a diez proveen una fuerte evidencia del valor diagnóstico, y para cocientes de probabilidad negativa, se sugiere que sea debajo de $0,1^{\left({ }^{(8)}\right.}$. Finalmente, se obtuvo el intervalo de confianza para ambos oídos. Se usó el paquete estadístico SPSS versión 22.

\section{ASPECTOS ÉTICOS}

Los procedimientos se aplicaron a los niños, previa autorización de los padres, con la firma del consentimiento informado. Se garantizó la confidencialidad de los datos obtenidos. El proyecto fue aprobado por el Comité de Ética Institucional del Instituto Nacional de Rehabilitación.

\section{RESULTADOS}

La población estuvo conformada por 117 niños, se excluyeron ocho niños en los que el familiar no pudo brindar datos completos, y otros trece niños por el antecedente de hiperbilirrubinemia neonatal. El total de participantes fue de 96 niños, de los cuales 55 eran de sexo masculino $(57,3 \%)$. La edad promedio de la evaluación fue de 13,28 meses ( $D E=9,58)$, mediana: 10,5 meses.

Según los factores de riesgo establecidos por la Joint Commitee on Infant Hearing ${ }^{(9)}$, dos pacientes $(2,1 \%)$ presentaron historia familiar de pérdida auditiva; diez $(10,4 \%)$ tuvieron anomalías craneofaciales; 31 (32,3\%) fueron derivados por la preocupación del cuidador con respecto al retraso del desarrollo del lenguaje, habla o audición; $12(12,5 \%)$ presentaron hallazgos físicos relacionado a otros síndromes que incluían pérdida neurosensorial o conductiva permanente; tres $(3,1 \%)$ con antecedente de infecciones posnatales asociadas a pérdida auditiva neurosensorial, y $38(39,6 \%)$ con cuidados neonatales especializados (Tabla 1). 
Tabla 1. Factores de riesgo relacionados a hipocuacusia

\begin{tabular}{lcc}
\hline Factor de riesgo & $\mathbf{n}$ & $\%$ \\
\hline $\begin{array}{l}\text { Historia familiar de pérdida auditiva (padres } \\
\text { sordos) }\end{array}$ & 2 & 2,1 \\
$\begin{array}{l}\text { Anomalías craneofaciales (fisura palatina, } \\
\text { disgenesia de pabellón auricular y } \\
\text { microcefalia) }\end{array}$ & 10 & 10,4 \\
$\begin{array}{l}\text { Preocupación del cuidador con respecto a } \\
\text { retraso en el desarrollo del lenguaje, habla } \\
\text { y/o audición. }\end{array}$ & 31 & 32,2 \\
$\begin{array}{l}\text { Hallazgos físicos asociado a otro síndrome } \\
\text { que incluya pérdida neurosensorial o }\end{array}$ & 12 & 12,5 \\
$\begin{array}{l}\text { conductiva permanente (síndrome de } \\
\text { Down y otitis media) }\end{array}$ & \\
$\begin{array}{l}\text { Infecciones postnatales asociadas con } \\
\text { pérdida auditiva neurosensorial (incluye } \\
\text { meningoencefalitis), sepsis neonatal }\end{array}$ & 3 & 3,1 \\
$\begin{array}{l}\text { Cuidados neonatales especializados } \\
\text { (asfixia perinatal, ictericia, incompatibilidad }\end{array}$ & 38 & 39,6 \\
$\begin{array}{l}\text { ABO, prematuridad, hidrocefalia, embarazo } \\
\text { múltiple, hipotiroidismo }\end{array}$ & & \\
\begin{tabular}{l} 
Total \\
\hline
\end{tabular} & 96 & 100 \\
\hline
\end{tabular}

En la Tabla 2 se muestra el valor promedio para los potenciales evocados auditivos de tronco cerebral; se encontró en el oído derecho, tanto para niños sanos como con antecedentes de riesgo, un valor de 34,1 dB y 44,3 dB respectivamente, y los valores obtenidos en el oído izquierdo fueron de $34,8 \mathrm{~dB}$ en niños sin factores de riesgo y 47,2 dB en niños con antecedente de riesgo.

En la Tabla 3 se muestra información sobre la prueba de otoemisiones acústicas; en el grupo de niños sanos, la mediana obtenida en el oído derecho e izquierdo fue de 5, sin diferencias en ambos oídos; en niños con antecedente de riesgo, la mediana del oído derecho fue 5 y en el oído izquierdo de 4 .

Como se muestra en la Figura 1, la curva ROC de las otoemisiones acústicas para el oído derecho el área debajo de la curva es de $0,830(p<0,001)$. En la Figura 2 se muestran las coordenadas para las otoemisiones acústicas del oído izquierdo, el área debajo de la curva es de 0,829 $(p<0,001)$.

Tabla 2. Potenciales evocados auditivos en niños sanos y con antecedente de riesgo para deficiencia auditiva

\begin{tabular}{lccc}
\hline & \multicolumn{2}{c}{$\begin{array}{c}\text { Potenciales evocados } \\
\text { auditivos de tronco } \\
\text { cerebral }\end{array}$} & \\
\cline { 2 - 3 } & \multicolumn{1}{c}{$\begin{array}{c}\text { Oído } \\
\text { derecho }\end{array}$} & $\begin{array}{c}\text { Oído } \\
\text { izquierdo }\end{array}$ & Valor $\boldsymbol{p}$ \\
\hline Niño sano & & & \\
\hline $\begin{array}{l}\text { Promedio (desviación } \\
\text { estándar) }\end{array}$ & $34,1(14,5)$ & $34,8(15,3)$ & 0,268 \\
\hline $\begin{array}{l}\text { Niño con antecedente } \\
\text { Promedio (desviación } \\
\text { estándar) }\end{array}$ & $44,3(24,9)$ & $47,2(26,1)$ & 0,058 \\
\hline
\end{tabular}

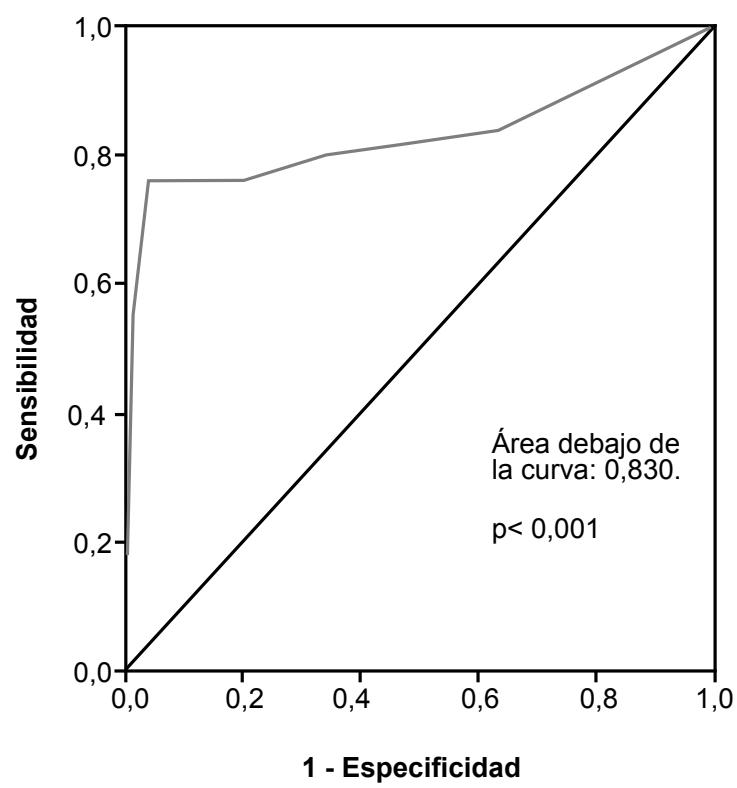

Figura 1. Coordenadas de la curva para las otoemisiones acústicas oído derecho

Si observamos la Tabla 4, que corresponde al oído derecho, la sensibilidad es de $76 \%$ en el oído derecho, el punto de corte es 1 que corresponde a 17,67 LR (+). Asimismo, si el paciente tiene como resultado $2 \mathrm{P}$ hay 7,6 veces la probabilidad y se considera sospechoso de alteración auditiva y se complementa la prueba con potenciales evocados auditivos de tronco cerebral. Hay que considerar los resultados de 3P como una probabilidad de 3,8 veces, de $4 \mathrm{P}$ con 2,33 veces y de $5 \mathrm{P}$ con 1,34 veces.

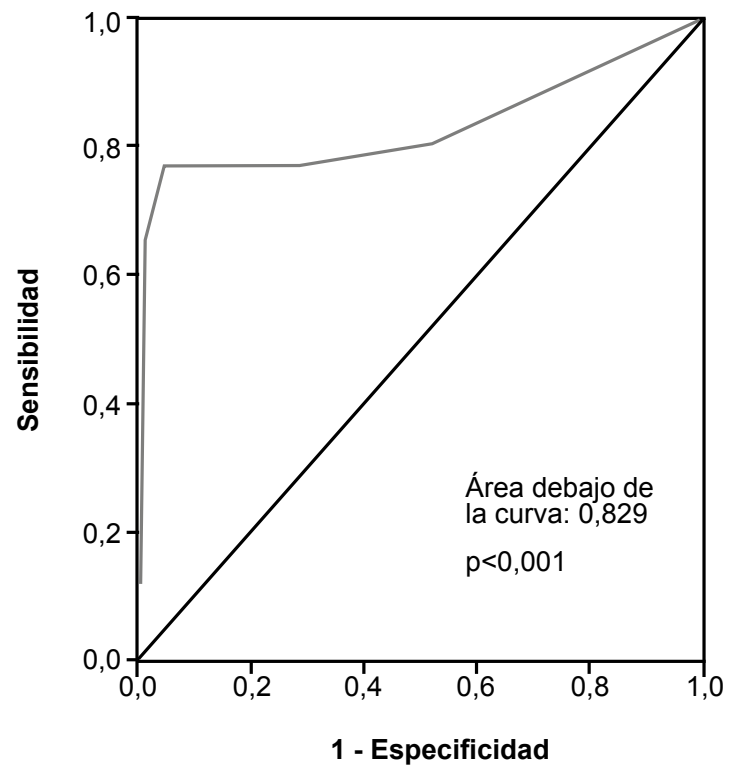

Figura 2. Coordenadas de la curva para las otoemisiones acústicas oído izquierdo 
Tabla 3. Otoemisiones acústicas en niños sanos y con antecedente de riesgo para deficiencia auditiva

\begin{tabular}{lccc}
\hline & \multicolumn{2}{c}{$\begin{array}{c}\text { Otoemisiones } \\
\text { acústicas }\end{array}$} & \\
\cline { 2 - 4 } & $\begin{array}{c}\text { Oído } \\
\text { derecho }\end{array}$ & $\begin{array}{c}\text { Oído } \\
\text { izquierdo }\end{array}$ & Valor $\boldsymbol{p}$ \\
\hline Niño sano & & & \\
\hline Mediana & 5 & 5 & 0,364 \\
\hline Percentil 25 & 4 & 3,5 & \\
\hline Percentil 75 & 6 & 5,5 & \\
Niño con antecedente & & & \\
\hline Mediana & 5 & 4 & 0,011 \\
\hline Percentil 25 & 1 & 1 & \\
\hline Percentil 75 & 6 & 5 & \\
\hline
\end{tabular}

En la Tabla 5, correspondiente al oído izquierdo, la sensibilidad es de $65 \%$, que coincide con la conformación de la curva ROC; el punto de corte es igual a 1, que corresponde al 16,72 de LR (+) que significa que hay 16 veces la probabilidad de que ante un resultado de emisión 1P el paciente tenga alteración auditiva. Asimismo, si el paciente tiene como resultado $2 \mathrm{P}$ hay una probabilidad de 8,36 veces, y se considera sospechoso de alteración auditiva y se complementa la prueba de emisiones otoacústicas con potenciales evocados auditivos de tronco cerebral. Hay que considerar los resultados de $3 \mathrm{P}$ con una probabilidad de 2,78 veces, de $4 \mathrm{P}$ con 1,54 veces y de $5 P$ 1,22 veces.

\section{DISCUSIÓN}

La percepción de estímulos sonoros inicia antes del nacimiento, lo cual implica la constante maduración del sistema auditivo. Dicha plasticidad se presenta hasta los cuatro años de edad ${ }^{(21)}$ por lo que se eligió este rango de edad en nuestro estudio.

Además, se cuenta con $67,7 \%$ de niños con antecedentes de riesgo importantes para la hipoacusia, es decir, en nuestro estudio predomina la población con factores de riesgo presentes. En relación a esa distribución de la población, Zivié ${ }^{(4)}$ menciona que, si bien las otoemisiones acústicas son altamente sensibles, hay un cierto número de niños que, por tener en su historia antecedentes de riesgo para hipoacusia, desarrollan lesión neuropática importante. Lo que significaría que las otoemisiones acústicas transitorias han ganado aceptación como una herramienta de cribado por sus ventajas como un test fiable, rápido y barato en relación a los PEATC.

En los pacientes con antecedentes de riesgo se debe complementar el resultado de la otoemisiones acústicas con los PEATC. Zivie obtuvo otoemisiones acústicas normales en el $92,5 \%$ de los neonatos a término, mientras que en los pretérmino solo se obtuvieron en $55,1 \%$. Es decir, en los niños pretérmino hay, además, otras complicaciones infecciosas y no infecciosas que agravan su estado. Por lo mencionado, las otoemisiones acústicas presentan limitaciones en la evaluación de la audición en neonatos hiperbilirrubinémicos, y en los que también están presentes otros factores de riesgo como la prematuridad, la asfixia perinatal, la incompatibilidad $A B O, y$ la sepsis neonatal.

En esta población existe una preocupación por los falsos negativos, tal y como sucede con el caso de la neuropatía auditiva o disincronía auditiva. Por lo tanto, en un cribado neonatal es recomendable, en estos casos, el uso combinado de PEATC y otoemisiones acústicas. La aplicación práctica de las especificaciones de los factores de riesgo de la $\mathrm{JCIH}$ y la CODEPEH (comisión para la detección de la hipoacusia infantil) requiere de una buena definición clínica ${ }^{(10,11)}$. Según Lirola ${ }^{(12)}$ las neuropatías auditivas adquiridas tienen un origen muy diverso. Pueden ser debidas a tóxicos, a alteraciones metabólicas como la diabetes o el hipotiroidismo, o a mecanismos inmunológicos como el Guillian-Barré. También se ha relacionado con procesos infecciosos, traumatismos craneales y la anoxia. La hiperbilirrubinemia y los estados de inmadurezprematuridad son etiologías en las que las neuropatías auditivas están presentes.

Tabla 4. Resultados de sensibilidad, especificidad, razón de verosimilitud positiva (LR+) y negativo (LR-)de oído derecho

\begin{tabular}{cccccc}
\hline $\begin{array}{c}\text { Positivo si es menor o } \\
\text { igual que }\end{array}$ & Sensibilidad & Especificidad & Sensibilidad+Especificidad & LR+ & LR- \\
\hline$-1,00$ & 0 & 1,00 & 1,00 & - & 1,00 \\
0,50 & 0,56 & 0,99 & 1,55 & 40,00 & 0,45 \\
1,50 & 0,76 & 0,96 & 1,72 & 17,67 & 0,25 \\
2,50 & 0,76 & 0,90 & 1,66 & 7,60 & 0,27 \\
3,50 & 0,76 & 0,80 & 1,56 & 3,80 & 0,30 \\
4,50 & 0,8 & 0,66 & 1,46 & 2,33 & 0,30 \\
5,50 & 0,84 & 0,37 & 1,21 & 1,34 & 0,43 \\
7,00 & 1 & 0,00 & 1,00 & 1,00 & - \\
\hline
\end{tabular}


Tabla 5.Resultados de sensibilidad, especificidad, razón de verosimilitud positiva $(L R+)$ y negativo (LR-) de oído izquierdo

\begin{tabular}{cccccc}
\hline $\begin{array}{c}\text { Positivo si es menor } \\
\text { o igual que }\end{array}$ & Sensibilidad & Especificidad & $\begin{array}{c}\text { sensibilidad } \\
\text { +especificidad }\end{array}$ & LR+ & LR- \\
\hline$-1,00$ & 0 & 1,00 & 1,00 & - & 1,00 \\
0,50 & 0,65 & 0,99 & 1,64 & 43,60 & 0,35 \\
1,50 & 0,77 & 0,95 & 1,72 & 16,72 & 0,24 \\
2,50 & 0,77 & 0,91 & 1,68 & 8,36 & 0,25 \\
3,50 & 0,77 & 0,72 & 1,49 & 2,78 & 0,32 \\
4,50 & 0,81 & 0,48 & 1,29 & 1,54 & 0,40 \\
5,50 & 0,88 & 0,28 & 1,16 & 1,22 & 0,42 \\
7,00 & 1 & 0,00 & 1,00 & 1,00 & - \\
\hline
\end{tabular}

En otros estudios como en el de Suppiej et al. ${ }^{(13)}$ también se han obtenido valores relacionados a la sensibilidad y especificidad, tanto de las otoemisiones acústicas y los PEATC realizados en recién nacidos con alto riesgo para hipoacusia. Explican que algunas diferencias entre los resultados de las dos pruebas pueden deberse a la presencia de factores de riesgo. Suppiej obtuvo $100 \%$ de sensibilidad y $78,2 \%$ de especificidad. Según la Academia Americana de Médicos de Familia la sensibilidad fue de $84 \%$ y la especificidad de $90 \%{ }^{(3)}$. Además, hay estudios en los que se transformaron los valores de especificidad y sensibilidad en coeficientes de probabilidad positiva ( $L R+$ ) y negativa (LR-). LR+ y LR- fueron 55,6 y 0,3 , respectivamente ${ }^{(12)}$.

Nuñez ${ }^{(10)}$ reportó que para la obtención de la sensibilidad y especificidad se retiraron los casos con ictericia para evitar la presencia de falsos negativos. No se cuenta con el grado de concentración de bilirrubina, por lo tanto, solo se colocó como ictericia grave cuando los familiares lo reportaron, o en la hoja de derivación se tenía dicha observación. Esto puede haber influenciado en el aumento o disminución de la capacidad diagnóstica del otoemisiones acústicas; es decir, muchas veces las estructuras que evalúa el OEA no se verían seriamente influenciadas, sin embargo, si pudiera haber afección a nivel de la vía auditiva que evalúa el PEATC.

Según Treviño ${ }^{(14)}$ un instrumento adecuado de tamizaje neonatal debería contar una sensibilidad por arriba de $97 \%$ para que exista un costo beneficio adecuado para el tamizaje universal. En México encontraron una sensibilidad del $90 \%$, más alta que el presente estudio. Esto nos lleva a explicar que, en estos casos lo que también ha sucedido en nuestro estudio, las emisiones otoacústicas no representarían una alternativa lo suficientemente discriminatoria como prueba de tamizaje. Se debería plantear, a futuro, un estudio con mayor población en la que se incluya mayor porcentaje de niños sin factores de riesgo para reevaluar la especificidad y sensibilidad de una prueba de tamizaje auditivo tan importante.
Silva (15), en un estudio de cohorte retrospectivo, incluyó a 125 pacientes mayores de 12 meses de edad, a quienes, por condiciones de alto riesgo para hipoacusia, se les realizó antes de los 6 meses de edad otoemisiones acústicas como prueba de tamizaje auditivo y obtuvieron una sensibilidad para sospechar hipoacusia neurosensorial del $82,5 \%$, y la especificidad estimada en su estudio fue de $90,9 \%$. Cabe mencionar que el porcentaje de pacientes con factores de riesgo relacionados a cuidados neonatales especializados fue de $16 \%$ en comparación de nuestro estudio que presentó $39,59 \%$ lo que podría estar relacionado con una mayor frecuencia de neuropatías auditivas. Además, las otoemisiones acústicas presentan también limitaciones para la detección de lesiones retrococleares y no detectan el umbral auditivo ${ }^{\left({ }^{(8)} \text {. }\right.}$

Algunas de las limitantes de este estudio son que los resultados no pueden ser extrapolados a los niños de todo el Perú, ya que el tamaño de muestra es pequeño y la mayor parte de nuestra población presentó factores de riesgo. Otra debilidad de nuestro estudio es que no se incluyó en de los procedimientos la timpanometría, de gran utilidad en el diagnóstico de las hipoacusias conductivas. Tampoco se incluyeron exámenes citogenéticos para explorar la etiología de los diferentes tipos de hipoacusia encontrados. Entre las fortalezas de este estudio se encuentra en ser el primero que se realiza en el Perú que involucra niños en edad donde definir el diagnóstico de hipoacusia va a asegurar, o no, el desarrollo normal del lenguaje oral.

El hallazgo del coeficiente de probabilidad de las otoemisiones acústicas en relación a los PEATC permitirá tener un primer indicio de deficiencia auditiva, pero, teniendo en cuenta los factores de riesgo que marcan la posibilidad de la presencia de la neuropatía auditiva.

En nuestro estudio a partir de la obtención de 2 "P", consideramos el resultado como dudoso. Por lo que no solamente se debe considerar el total de la prueba como "pasar" o "a control" sino que se debe analizar la 
cantidad de $\mathrm{P}$ por oído. Existe la teoría de que el oído derecho es mejor que el oído izquierdo. La asimetría en las otoemisiones acústicas puede ser producto del sistema eferente ${ }^{(16)}$, lo que se ha obtenido en el presente trabajo justamente es esta relación, basada en los resultados obtenidos en los promedios de los PEATC por oído y el valor de la mediana de la otoemisiones acústicas por oído.

De acuerdo con Trinidad (17) la edad es un parámetro determinante que influye en la necesidad de repetir exploraciones. La edad óptima para la realización de la prueba es menor de 30 días. En el presente estudio, el promedio de edad fue de 13,28 meses, por lo que se considera que la cobertura lograda fue baja comparada con otros estudios representativos ${ }^{(18,19)}$. La calidad de los programas de detección precoz de hipoacusia es el índice de cobertura que valora el número de pacientes menores de tres meses incluidos en el tamizaje ${ }^{(20,21)}$.

En conclusión, las emisiones otoacústicas no representarían una alternativa lo suficientemente discriminatoria como prueba de tamizaje en niños con factores de riesgo. Por lo que, en estos casos, se deben realizar PEATC. Se debería plantear, a futuro, un estudio con mayor población en la que se incluya mayor porcentaje de niños sin factores de riesgo, para reevaluar la especificidad y sensibilidad de una prueba de tamizaje auditivo tan importante. Además, se remarca la importancia de analizar el número de "P" obtenidas en el resultado de las otoemisiones acústicas.

Fuentes de financiamiento: Instituto Nacional de Rehabilitación "Dra. Adriana Rebaza Flores" Amistad PerúJapón. Lima, Perú (INR) - MINSA.

\section{Conflictos de interés: ninguna.}

Contribuciones de autoría: JRT, JAA han participado en la concepción y diseño del artículo, recolección de resultados, análisis e interpretación de datos y redacción del artículo. Además, JRT realizó la revisión crítica del artículo, la aprobación de su versión final, coordinó en relación al aporte de pacientes o material de estudio, asesoría estadística, fuente de financiamiento y asesoría técnica.

\section{REFERENCIAS BIBLIOGRÁFICAS}

1. Campos M, López-Campos D, Perez B, López-Aguado D. Correlación entre emisiones otoacústicas y PETC. La importancia de su empleo combinado. Acta Otorrinolaringol Esp 2003; 54(10): 667-670.

2. Ramos-Trinidad G, Pando-Pinto J, Vega-Cuadri A, Serrano-Berrocal M, Trinidad-Ruiz G, Blasco-Huelva A. Detección Precoz de Hipoacusia en Recién Nacidos mediante otoemisiones acústicas evocadas transitorias. An Esp Pediatr 1999;50(2):166-171.

3. Yousefi J, Ajalloueyan M, Amirsalari S, Hassanali-Fard M. The Specificity and sensitivity of transient otoacustic emission in neonatal hearing screening compared with diagnostic test of auditory brain stem response in Tehran Hospitals. Iran J Pediatr Apr 2013; 23(2): 199-204.

4. Zivić L, Obradović S, Stojanović S, Zbiljić I, Jakovljević VL, Zivić D et al. Neonatal screening of hearing function by otoacustic emissions- a single center experience. Vojnosanit Pregl 2012 Apr;69(4):340-4.

5. The Eclipse Platform Operation Manual for EP15, EP25, TEOAE25, DPOAE20, ABRIS, ASSR. Interacoustics. Quick Guides.

6. OtoRead Instructions for use, Interacoustics.
7. Aguilar Bolaños M, Flores Pérez J, Martínez Haro M, (2014) Detección temprana de hipoacusia en población infantil de Tlaxcala. Rev Mex AMCAOF 2014; 3(2): 49-53.

8. Deeks JJ. Systematic reviews of evaluations of diagnostic and screening tests. BMJ. 2001 Jul 21;323(7305):15762.

9. Denia Lafuente A, Lombardero Pozas B. Detección, diagnóstico y tratamiento precoz de la sordera en la infancia. Actas del Simposio Internacional celebrado en la Fundación Ramón Areces. Editorial Centro de Estudios Ramón Areces. Madrid. 2009.

10. Núñez-Batalla F, Carro-Fernández P, Antuña-León ME, GonzálezTrelles T. Incidence of hypoacusia secondary to hyperbilirubinaemia in a universal neonatal auditory screening programme based on otoacoustic emissions and evoked auditory potentials. Acta Otorrinolaringol Esp. 2008 Mar;59(3):108-13.

11. Rodriguez-Dominguez F, CubillanaHerrero J, Cañizares-Gallardo N, Perez-Aguilera R, Prevalencia de la Neuropatía auditiva: estudio prospectivo en un hospital de tercer nivel. Acta Otorrinolaringol Esp. 2007 Jun-Jul;58(6):239-45.
12. Lirola-Delgado A. Neuropatías Auditivas. Rev Soc Otorrinolaringol Castilla Leon Cantab La Rioja 2014; 5(25): 207-211.

13. Suppiej A, Rizzardi E, Zanardo V, Franzoi M, Ermani M, Orzan E. Reliability of hearing screening in high risk neonates: comparative study of otoacoustic emission, automated and convencional auditory brainstem response. Clinical Neurophysiology 2007; 118: 869-876.

14. Treviño-González JL, Santos-Lartigue R, Marroquin-Escamilla AR, AbregoMoya V, Villagómez-Ortíz J, GonzálezAndrade B. Tamizaje auditivo en recién nacidos del Hospital Universitario Dr. José E. González. Medicina Universitaria 2011;13(52):139-143

15. Silva Rueda R. , Ordoñez Ordoñez L, Díaz Patiño D, Gonzales Marín N. Tamizaje Auditivo neonatal en pacientes de alto riesgo con otoemisiones acústicas: evaluación de resultados. Universidad Militar Nueva Granada.

16. Keogh T, Kei J, Driscoll C, Smyth V. Distortion-Product Otoacoustic Emissions in Schoolchildren: Effects of, and Gender. J Am Acad Audiol 2001; Nov-Dec;12(10):506-13. 
17. Trinidad G. Técnicas de screening de la audición: programa de detección precoz de sorderas con otoemisiones evocadas transitorias (OEAT). En: Prof. Marco J, Matéu S, coordinadores. Libro blanco sobre hipoacusia: detección precoz de la hipoacusia en recién nacidos. Madrid: CODEPEH, Ministerio de Sanidad y Consumo; 2003. P. 45-88.

18. Benito-Orejas JI, Ramírez-Cano B, Morais-Pérez D, Fernández-Calvo JL, Almaraz-Gómez A. Results of applying protocol for early detection of hypoacusia in newborn infant for 42 month. Acta Otorrinolaringol Esp. $2008 \mathrm{Mar}$;59(3):96-101.

19. Sandoval-García M, Iglesias-Leboreiro J, Silva-Ramírez H, Frid-Chernitzky J, Rendón-Macías ME. Frecuencia de hipoacusia neonatal en un hospital privado. Tamiz Auditivo. Rev Mex Pediatr 2012; 79(4); 174-178

20. Gonzáles de Dios J, Mollar Maseres J, Rebagliato-Russo M. Evaluación de un Programa de Detección Precoz de la Hipoacusia en el recién nacido. An
Pediatr 2005;63:230-7 - Vol. 63 Núm.3 DOI: $10.1157 / 13078486$

21. Urdiales J, Álvaro E, López-Fernández I, Vázquez Casares G, PiqueroFernández J, Conde-López $\mathrm{M}$, et al. Revisión de los métodos de screening en hipoacusias. Bol. Pediatr. 2003; 43(185): 272-280.

Correspondencia: Rado- Triveño Julia. Dirección: Jirón Juan de Rada 161 Dpto. 101 Urbanización Liguria. Surco.

Teléfono: 999335160.

Correo electrónico:juliarado@hotmail.com
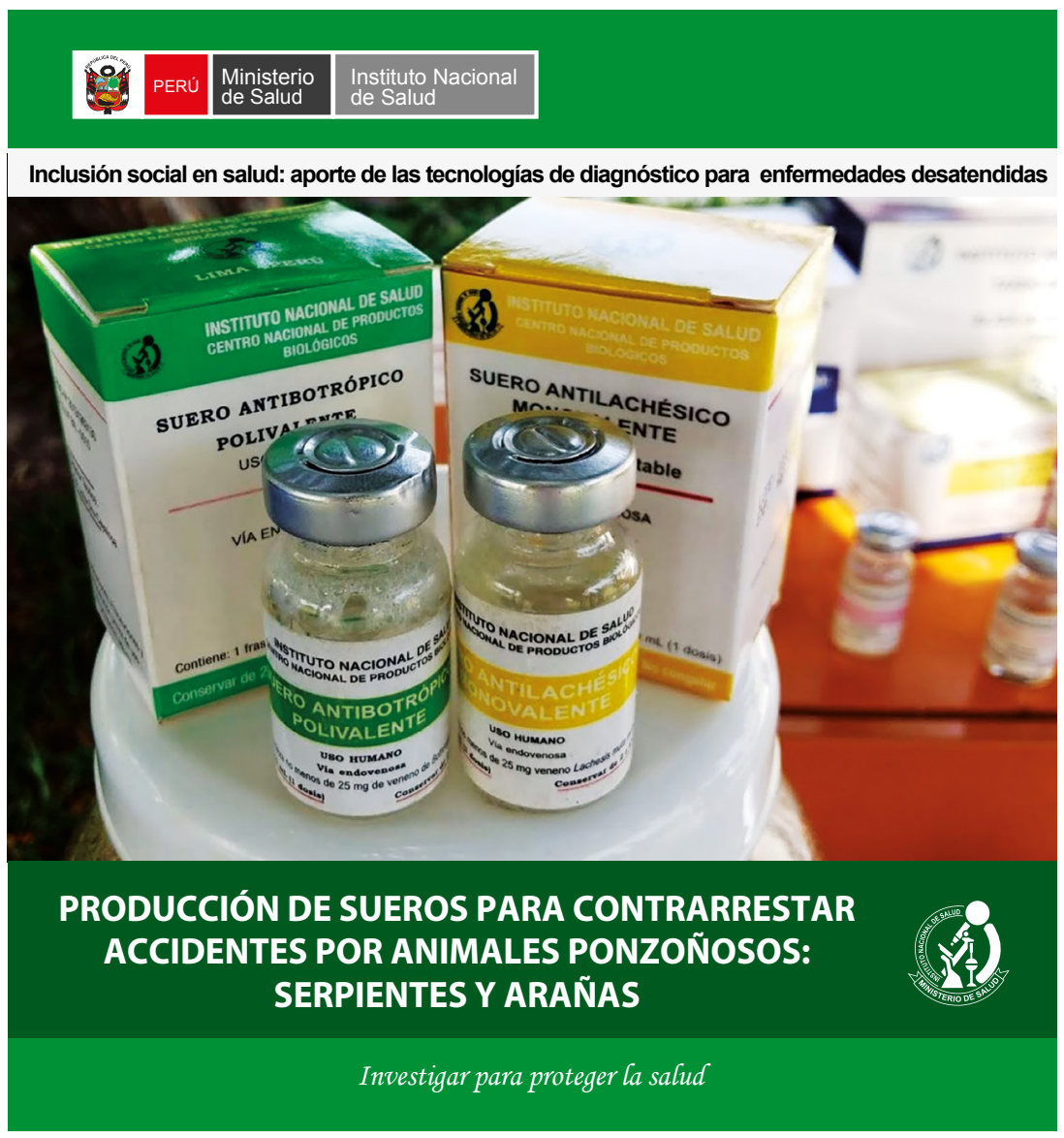